\title{
From quantum groups to genetic mutations
}

\author{
A. Sciarrino \\ Dipartimento di Scienze Fisiche, Università di Napoli "Federico II" \\ I.N.F.N., Sezione di Napoli \\ Complesso Universitario di Monte S. Angelo \\ Via Cintia, I-80126 Napoli, Italy
}

\begin{abstract}
In the framework of the crystal basis model of the genetic code, where each codon is assigned to an irreducible representation of $U_{q \rightarrow 0}(s l(2) \oplus \operatorname{sl}(2))$, single base mutation matrices are introduced. The strength of the mutation is assumed to depend on the "distance" between the codons. Preliminary general predictions of the model are compared with experimental data, with a satisfactory agreement.
\end{abstract}

Symmetries in Science XIII

Bregenz, July 20-24 2003 



\section{Introduction}

Among the numerous and important questions offered to the theoretical physicist by the sciences of life, the ones relative to the genetic code present a particular interest. The DNA structure and the mechanism of polypeptid fixation from codons possess appealing aspects for the theorist and, indeed, the first proposal of genetic code may be ascribed to G. Gamow [1] in 1954, less than year after the discovery of DNA by Watson and Crick. Let us briefly recall some essential features, see e.g. [2]. First the DNA macromolecule is constituted by two linear chains of nucleotides in a double helix shape. There are four different nucleotides, characterised by their bases: adenine (A) and guanine $(\mathrm{G})$ (purines family), cytosine $(\mathrm{C})$ and thymine $(\mathrm{T})$ (pyrimidines family). Note also that an A (resp. T) base in one strand is connected with two hydrogen bonds to a $\mathrm{T}$ (resp. A) base in the other strand, while a $\mathrm{C}$ (resp. $\mathrm{G}$ ) base is related to a $\mathrm{G}$ (resp. C) base with three hydrogen bonds. The genetic information is transmitted via the messenger ribonucleic acid or mRNA. During this operation, called transcription, the A, G, C, T bases in one strand of the DNA are associated respectively to the $\mathrm{U}, \mathrm{C}, \mathrm{G}, \mathrm{A}$ bases, (U denoting the uracile base) of RNA. Then, a triplet of nucleotides or codon will be related to an amino-acid. More precisely, a codon is defined as an ordered sequence of three nucleotides, e.g. AAG, AGA and GAA, and one enumerates in this way $4 \times 4 \times 4=64$ different codons. In the universal eukariotic code (see Table 3), 61 of such triplets encode the amino-acids, while the three codons UAA, UAG and UGA, which are called non-sense or stop-codons, play the role to stop the biosynthesis process. Indeed, the genetic code is the association between codons and amino-acids. But since one distinguishes only 20 amino-acids ${ }^{1}$ related to the 61 codons, it follows that the genetic code is degenerated. From Table 3, one remarks the presence of 3 sextets, 5 quadruplets, 1 triplet, 9 doublets and 2 singlets of codons, each multiplet corresponding to a specific amino-acid. Since its appearance on the earth life has been characterized by its continuous change. Spontaneous genetic mutations, i.e. modifications of the DNA genomic sequences, play a fundamental role in the evolution. In the present paper I only deal with point mutations, that is with single base (single nucleotide) changes. More generally, mutations include changes of more than one nucleotide, insertions and deletions of nucleotides, frame-shifts and inversions. The point mutations are usually modeled by stationary, homogeneous Markov process, which assume:

1) the nucleotide positions are stochastically independent one from another, which is clearly not true in functional sequences;

2) the mutation is not depending on the site and constant in time, which ignores the existence of "hot spots" for mutations as well as the probable existence of evolutionary spurts;

3) the nucleotide frequencies are equilibrium frequencies. Moreover a common belief is that tha change of the $3 r d$ nucleotide is more frequent than the change of the 1st nucleotide, the latter being more frequent than the change of the second one

In the following the labels $i, j$ run in the set analysed, e.g. $i, j \in\{C, T, G, A\}$ ( $T$ being replaced by $U$ in RNA) for single nucleotides changes or $i, j$ run in a 20-dim set for the amino-acids substitution matrix or in a 64-dim set for for the codon substitution matrix. The transition matrix $\mathbf{Q}$, where $Q_{i j}>0(i \neq j)$ represents the transition rate between the $j$ state and the $i$ state, in the choosen unit

\footnotetext{
${ }^{1}$ Alanine (Ala), Arginine (Arg), Asparagine (Asn), Aspartic acid (Asp), Cysteine (Cys), Glutamine (Gln), Glutamic acid (Glu), Glycine (Gly), Histidine (His), Isoleucine (Ile), Leucine (Leu), Lysine (Lys), Methionine (Met), Phenylalanine (Phe), Proline (Pro), Serine (Ser), Threonine (Thr), Tryptophane (Trp), Tyrosine (Tyr), Valine (Val).
} 
of "time", and it is normalised to

$$
0>Q_{i i}=1-\sum_{j \neq i} Q_{i j}
$$

The evolution matrix $\mathbf{P}$, where $P_{i j}(t)$ gives the probability that the $j$ state at time $t=0$, will be replaced, at time $t$, by the $i$ state, satisfies the differential equation

$$
\frac{d P_{i j}(t)}{d t}=\sum_{k} P_{i k}(t) Q_{k j} \quad \Leftrightarrow \quad \mathbf{P}(t)=\mathbf{P}(0) \exp \mathbf{Q} t \quad \mathbf{P}(0)=\mathbf{1}
$$

In the Markov model, with discretized time $\tau$, we have

$$
\mathbf{P}((n+1) \tau)=\mathbf{Q} \mathbf{P}(n \tau)
$$

The most simple reversible model describing single nucleotide changes depends on 1 parameter and the most complex not reversible model depends on 12 parameters [3]. ${ }^{2}$ These models consider the DNA sequences as set of nucleotides each nucleotide evolving independently of the others; they are not able to make, a priori, any prediction on the reversibility of a mutation and naturally predict that a nucleotide change happens at the same rate independently of which codon it belongs to. The following shortcomings are particularly serious: the Markov models are indeed unable to explain i) the dependence of mutations on the nature of the neighbouring nucleotides [ $[5]$. These features can of course be accounted introducing more new unknown parameters or new type of models, see [6];

ii) the fact that mutations occur more frequently between amino acids with similar physico-chemical properties, which generally have similar functional roles. Generally in the literature it is stated that the nature of the 2nd nucleotide strongly determines the physico-chemical propertie. In the seventies Konopolchenko and Rumer [7] have remarked that amino acids with similar physico-chemical properties can be described by assigning a suitable charge $Q$ to the first dinucleotide (called "root" by the authors) of the codon, in particular "strong roots" ("weak roots'), corresponding to multiplets of codons of dimension $4(\leq 3)$, have $Q>0(Q<0)$. Note that sextets appear as the sum of a quartet and of a doublet.

The aim of this paper is to propose a model in which the strength of the mutation depends on a suitably defined distance between codons. This model reduces to the Markov model if the distance dependence is assumed constant, but it is able, in principle, to take into some account the points i)-ii). The first requirement to build such a model is to identify codons as mathematical objects, in particular as vectors in a suitable space. This will be done in the framework of the crystal basis model of the genetic code [8]. In this model the 4 nucleotides are assigned to the (4-dim fundamental) irreducible representation (irrep.) $(1 / 2,1 / 2)$ of $U_{q \rightarrow 0}(s l(2) \oplus \operatorname{sl}(2))$ with the following assignment for the values of the third component of $\vec{J}$ for the two $\operatorname{sl}(2)$ which in the following will be denoted as $s l_{H}(2)$ and $s l_{V}(2)$ :

$$
\mathrm{C} \equiv\left(+\frac{1}{2},+\frac{1}{2}\right) \quad \mathrm{T} / \mathrm{U} \equiv\left(-\frac{1}{2},+\frac{1}{2}\right) \quad \mathrm{G} \equiv\left(+\frac{1}{2},-\frac{1}{2}\right) \quad \mathrm{A} \equiv\left(-\frac{1}{2},-\frac{1}{2}\right)
$$

and the codons, triple of nucleotides, to the 3 -fold tensor product of $(1 / 2,1 / 2)$. The assignment of the codons to the different irreps. and the correspondence with the encoded amino acid in the eukaryotic code is provided in Table 3. Let us emphasize that the assignment of the codons to the different irreps. is a straightforward consequence of the assumed labelling of the nucleotides eq.(44) and of the

\footnotetext{
${ }^{2}$ For a review of the different Markov models with a large list of the original papers, see Cap. 3 of $\llbracket$.
} 
Kashiwara's theorem on the tensor product of irreps. in the crystal basis [9]. In the following we call nearest codon codons differing by only one nucleotide. The effects of a single nucleotide mutation in the codons are represented, neglecting the mutations into or from the three stop codons which are not detectable in the considered set of experimental data, by a $61 x 61$ (symmetric) matrix, whose elements, in first approximation, will be assumed vanishing if non connecting nearest codons.

In ref. [10] it has been shown that amino acids with similar properties can grouped together looking to the content of the irrep. of the first dinucleotide (or "root"), in particular to the values of the charge $Q$ and the third generator of $s_{V}(2)$. The charge $Q$ can be expressed as ${ }^{3}$

$$
Q=4 J_{3, H}+C_{V}\left(J_{3, V}+1\right)-1
$$

In that paper the analysis has been performed for 10 physico-chemical properties: the Chou-Fasman conformational parameters, which give a measure of the probability of the amino acid to form respectively a helix, a sheet and a turn; the Grantham polarity; the relative hydrophilicity; the thermodynamic activation parameters at $298 \mathrm{~K}: \Delta H$ (enthalpy, in $\mathrm{kJ} / \mathrm{mol}$ ), $\Delta G$ (free energy, in $\mathrm{kJ} / \mathrm{mol}$ ) and $\Delta S$ (entropy, in $\mathrm{J} / \mathrm{mole} / \mathrm{K}$ ); the dissociation constants at $298 \mathrm{~K}$; the isoelectronic point, i.e. the $p H$ value at which no electrophoresis occurs. The strength of the mutation inducing operator is assumed to depend on the distance between the initial codon and the final codon, i.e. the codon appearing as result of the mutation. In the literature many attempts to define distance between codons exist based on the similarity of their physico-chemical properties or of those of the encoded amino-acid. Sometimes the distance between amino acids is defined by the strength of their mutation. Here I follow a completely different approach as I define a priori a distance and then I try to derive the strength of their mutation.

\section{The mutation matrix}

In order to be able to define the distance we make a correspondence between a codon and a point in $n$-dim. Euclidean space. For sake of simplicity, presently we assume a 1-dim space. ${ }^{4}$ The correspondence between codons and real numbers is realized through the eigenvalues of the following operator

$$
\hat{X}=\left[\alpha Q^{1}-\beta J_{3, V}^{1}\left(J_{3, V}^{1}-1\right)+4 \gamma\left(C_{H}+C_{V}\right)\right] 2\left(J_{3, H}+\eta J_{3, V}\right)
$$

where $\alpha, \beta, \gamma$ and $\eta$ are real positive parameters $(\eta>1$ as mutations between pyrimidines and purines (tranversions, $\Delta J_{3, V} \neq 0$ ) occur less frequently than mutations between pyrimidines or purines (transitions $\left.\Delta J_{3, H} \neq 0\right)$ ); $Q^{1}$ and $J_{3, V}^{1}$ are, respectively, the "charge", given by eq.(5), and the third generators of $s l_{V}(2)$ of the first dinucleotide of the codon $X Y Z$, that is $X Y$, and $C_{H}, J_{3, H}$ (resp. $\left.C_{V}, J_{3, V}\right)$ are the Casimir operator and the third generator of $s l_{H}(2)$ (resp. $s l_{V}(2)$ ) for the trinucleotide state or codon,

$$
\hat{X} \psi(X Y Z)=r(X Y Z) \psi(X Y Z)
$$

where $\psi(X Y Z)$ is the state $\in V, V$ being the space of the irreps. of $U_{q \rightarrow 0}\left(s l_{H}(2) \oplus s l_{V}(2)\right)$, corresponding to the XYZ codon and, using the same notation for the operators and for their eigenvalues,

$$
r=\left[\alpha Q^{1}-\beta J_{3, V}^{1}\left(J_{3, V}^{1}-1\right)+4 \gamma\left(C_{H}+C_{V}\right)\right] 2\left(J_{3, H}+\eta J_{3, V}\right)
$$

the values of the quantities appearing in eq.(8) are given in Table 1 and Table 3. The transition

\footnotetext{
${ }^{3}$ Note that the numerical values of eq.(5) are slightly different from those of [7.

${ }^{4}$ The use of a 2 -dim space, related to the roots of the two commuting $s l(2)$, may seem the most naturale choice.
} 
Table 1: Dinucleotides representation content and charge Q

\begin{tabular}{crrrrccrrrrc}
\hline dimer & $J_{H}$ & $J_{V}$ & $J_{3 H}$ & $J_{3 V}$ & $Q$ & dimer & $J_{H}$ & $J_{V}$ & $J_{3 H}$ & $J_{3 V}$ & $Q$ \\
\hline CC & 1 & 1 & 1 & 1 & 7 & GC & 1 & 1 & 1 & 0 & 5 \\
CU & 0 & 1 & 0 & 1 & 1 & GU & 0 & 1 & 0 & 0 & 1 \\
CG & 1 & 0 & 1 & 0 & 3 & GG & 1 & 1 & 1 & -1 & 3 \\
CA & 0 & 0 & 0 & 0 & -1 & GA & 0 & 1 & 0 & -1 & -1 \\
UC & 1 & 1 & 0 & 1 & 3 & AC & 1 & 1 & 0 & 0 & 1 \\
UU & 1 & 1 & -1 & 1 & -1 & AU & 1 & 1 & -1 & 0 & -3 \\
UG & 1 & 0 & 0 & 0 & -1 & AG & 1 & 1 & 0 & -1 & -1 \\
UA & 1 & 0 & -1 & 0 & -5 & AA & 1 & 1 & -1 & -1 & -5 \\
\hline
\end{tabular}

matrix between the codon $i=X Y Z$ and the codon $j=X^{\prime} Y^{\prime} Z^{\prime}$ is

$$
Q_{j i}=F\left(d_{j i}\right) q_{j i} \quad j \neq i
$$

where $F\left(d_{j i}\right)$, the strength of the transition, is a decreasing function of the argument and $d_{j i}$ is the distance between the initial and final codon

$$
d_{j i}=\left|r\left(X^{\prime} Y^{\prime} Z^{\prime}\right)-r(X Y Z)\right|
$$

and $q_{j i}$ is the element of a matrix $\mathbf{q}$ such that

$$
q_{j i}=1 \quad \mathrm{i}, \mathrm{j} \text { nearest codons } \quad q_{j i}=0 \quad \text { otherwise }
$$

If the strength are considered as constants, our model is essentially equivalent to a reversible Markov model with constant parameters. A few words to justify the assumptions eq.(8). Of course there is an arbitrary infinite way of defining the correspondence between a codon and a point of an Euclidean space. Our choice is such that to a larger variation of the charge, i.e. to a larger variation of the physico-chemical properties, corresponds a larger distance and that the distance between codons in the same irrep. is lower that codon in differents irreps.. Generally, from eq.(88), the distance between two codons, differing by a nucleotide in the middle position or in the first position, is larger, due to the change of the value of the charge, than the distance between two codons, differing by a nucleotide in the third position. At this stage our model can be considered as a markovian model with neighbors depending parameters.

\section{Amino acid substitution matrices}

In this section we recall the definition and the differences between the experimentally determined mutation matrix. The sequences alignment of proteins is a most powerful tool to get insights on the protein functions and to compute substitution rates due to evolutionary processes. The first scheme was proposed in the seventies by M. Dayhoff [11] and it is generally considered as the standard scheme. It is based on the alignments of protein sequences that are at least $85 \%$ identical. The evolutionary distance in measured in "accepted point mutation" (PAM). Two sequences are said to be 1 PAM distant if they differ on average by one accepted-point mutation per 100 amino acids. The term "accepted" means that the mutation of the amino acid has been incorporated into the protein's progeny, i.e. the mutation has not produced harmful consequences. The original Dayhoff matrix, by construction, was biased by the sample of proteins available at that time, mainly small 
globular preoteins, and emphasized the rate of mutation in the highly mutable amino acids. Another shortcoming of this scheme is that relationships between far distant sequences are poorly inferred, due to to the presence of deletions and insertions. A matrix, taking into account substitutions poorly represented in the original Dayhoff's analysis and making use of a statistics about 35 times higher, was computed in ref. [12 and it is known as PET91. ${ }^{5}$ We make a comparison between our data and the 1-PAM PET91 matrix, see Table II of [12]. In that table the data are referred to the substitution of the amino acids, so we cannot compare them directly with our predictions, which refer to the codon mutations. We have to consider for each amino acid the multiplet of codons encoding it and then to consider only the one-nucleotide mutations. In this process we have to take into account the preferred codon usages, which depend on the biological species and on the type of gene analysed. In this preliminary analysis we make the simple (and definitely incorrect) assumption of an uniform codon usage. The experimental Dayhoff matrix entries between the amino acids $a$ and $b$ are identified as

$$
M_{a b}=\sum_{i, j} f_{i}^{a} M_{a b}^{i j}
$$

where $f_{i}^{a}$ is the frequence of the $i$ codon in the amino acid $a, M_{a b}^{i j}$ is the substitution rate matrix for the codons and the sum is over all the codons encoding the amino acids $a$ and $b$ differing by only one nucleotide. The comparison with experimental data requires one more assumption. We have to compare the matrix

$$
\mathbf{P}(t)=\exp \mathbf{Q} t
$$

with the $x-P A M$-mutation matrix $\mathbf{M}_{x-P A M}$ which is computed at a $x$ distance between the amino acids sequences. Commonly $1-P A M$ evolutionary distance is considered to correspond to a time interval of $\approx 1 \times 10^{7}$ years and the correspondence between the PAM matrix and the instantaneous rate matrix is

$$
\mathbf{M}_{1-P A M}=\exp \mathbf{Q} t \approx \mathbf{1}+0.1 \mathbf{Q}
$$

i.e. the unit of time is choosen $\tau_{0}=1 \times 10^{8}$ years. It should be remarked that the above matrices, by construction are really divergence matrices, that is they provide the probability that the $j$ state in the first sequence, will be replaced by the $i$ state in the second xPAM distant sequence. Moreover these matrices have been build up assuming a symmetric probabily of mutation between two amino acids and, consequently, the estimated rate is lower for the amino acid which has a larger frequency. Therefore, strictly speaking, a direct comparison between the rate matrix eq.(9) and the amino acid substitution matrices is uncorrect. However, as in the present work we present only semiquantitative comparison, our conclusions should not be sensibly affected by the above remarks.

\section{Predictions of model}

\subsection{Stability}

From the assignment of the codons to the different irreps., see Table 3, and the assumed distance, see eq.(10), we can make a set of general predictions independent of the structure of the $F$ function and of the detailed values of $\alpha, \beta, \gamma$ and $\eta$. Considering a single-nucleotide mutation, each codon can make transition in the (9) nearest codons. Some of these codons can be synonimous (silent mutations)

\footnotetext{
${ }^{5}$ To study the relations for distant sequences a more reliable model has been proposed in 1992 [13], which is presently known as block substitution matrix (BLOSUM).
} 
or stop codons (nonsense mutations), both being unobservable in the framework of the substitution matrices. However, without a thorough analysis of their physico-chemical properties and/or their functional functions, we should expect amino acids encoded by multiplets of the same dimension to be approximately equally stable, i.e. the diagonal entries of the mutation matrix $\mathbf{M}$ should be of the same order. In the crystal basis model, see Table 3, not all the codons are on the same foot as they belong to different irreps. spaces. We indeed expect that mutations between codons in the same irrep. to occur more frequently than mutations between different irreps., provided that the values of $J_{3, V}^{1}$ are close and the signs of their charge $Q$ are the same. This requires that we have to compare respectively long multiplets and short multiplets between them. Moreover in each fixed space, the codons represented by highest or lowest weight are "surrounded" by a smaller number of nearest codons. From an analysis of the positions of the codons in the different irreps., we can qualitatively, from eq.(8), derive a hierarchy in the stability.

$$
\begin{gathered}
\text { Gly }>\text { Pro }>\text { Ala }>\text { Thr }>\text { Ser } * \\
\text { Phe }>\text { LysI }>l e * *>A s n \\
\text { Leu* }>\text { Val Glu }>\text { Asp } \\
\text { His } \approx \text { Gln Trp }>>\text { Met }
\end{gathered}
$$

where the ${ }^{*}(* *)$ is written to recall that we are dealing with a sextet (triplet), so our analysis is less reliable. A comparison with the experimental data from the PET91 and Dayhoff matrices for the average mutability, see Table 2, shows a remarkably satisfactory aggreement (higher stability implies lower mutability). Note that the comparison between His and Gln which, at first sight, is not satisfactory with the Dayhoff data, should be analyzed on the light of the wide range of variation of the values of the average relative mutability for the doublets (between 20 and 134). A more detailed analysis should require an evaluation of the form of the $F$ functions and of the values of the constants appearing in eq.(8).

Table 2: Relative mutabiliity for the 20 amino acids with respect to Ala, arbitrarily fixed to 100 , from Table III of [12].

\begin{tabular}{crrrrc}
\hline amino acid & PET91 & Dayhoff & amino acid & PET91 & Dayhoff \\
\hline Ala & 100 & 100 & Leu & 54 & 40 \\
Arg & 83 & 65 & Lys & 72 & 56 \\
Asn & 104 & 134 & Met & 93 & 94 \\
Asp & 86 & 106 & Phe & 51 & 41 \\
Cys & 44 & 20 & Pro & 58 & 56 \\
Gln & 84 & 93 & Ser & 117 & 120 \\
Glu & 77 & 102 & Thr & 107 & 97 \\
Gly & 50 & 49 & Trp & 25 & 18 \\
His & 91 & 66 & Tyr & 50 & 41 \\
Ile & 103 & 96 & Val & 98 & 74 \\
\hline
\end{tabular}

\subsection{Relation between rates}

In the following we use the standard notation $\mathrm{Y}=\mathrm{C}, \mathrm{U}$ (pyrimidines) and $\mathrm{R}=\mathrm{G}, \mathrm{A}$ (purines) and $\mathrm{N}$ for any nucleotide. First we look for qualitative prediction for the rate of transition between two amino acids $a$ and $b(\mathrm{R}(a \Leftrightarrow b))$ which follow directly from eq.(91) and from the assumed behaviour 
of the $F$ function, without any information of the values of $\alpha, \beta, \gamma$. Fron an inspection of eqs.(10), (18), (5) and Tables 1, 3. we can write a set of inequalities between the rates for several amino acids. The results of our analysis are reported in Table 4 where for any couple of amino acids we write the experimental values (Exp) taken from PET matrix [12. Of course we cannot make any more precise statement on the range of the inequalities, due to the yet undefined $F$ function. From the experimental data that $R(P h e \Leftrightarrow$ Leu $)>R(P h e \Leftrightarrow$ Tyr $)($ Exp.: $230-179)$ we derive $\eta>2$. Then we expect

$$
R(\text { Ala } \Leftrightarrow \operatorname{Pro})<R(\text { Ala } \Leftrightarrow \text { Val }) \quad \text { Exp: } \quad 23-193
$$

Let we remark that the following mutations between doublets: Asn $\Leftrightarrow$ Lys (AAY $\Leftrightarrow$ AAR), Asp $\Leftrightarrow$ Glu (GAY $\Leftrightarrow \mathrm{GAR}), H i s \Leftrightarrow G \ln (\mathrm{CAY} \Leftrightarrow \mathrm{CAR}$ ), share the common features to involve a mutation in the 3rd nucleotide and to have the same 2nd nucleotide A. So from the assumption that the middle nucleotide is the one which strongly determines the physico-chemical properties, comparable mutation rates should be expected. On the contrary in our model, from eq. (8), we expect different rates, except for a numerical coincidence for at most two of the considered mutations. The experimental rates are different (resp.: $150-478-233$ ). So we derive the following inequality:

$$
|60 \gamma-4 \beta-10 \alpha|>|12 \gamma-2 \alpha|>|36 \gamma-4 \beta-2 \alpha|
$$

Let us note that our analysis puts into evidence:

a) a dissimilarity between the transversions $C \Leftrightarrow A$ and $U \Leftrightarrow G$, which apparently has not before either remarked;

b) a "penalty", in the form of an increase of the distance, appears for mutations between codons with $\left|J_{3, H}\right|$ or $\left|J_{3, V}\right|>1 / 2$.

Let us recall once more that in the determination of the mutation rate the mutability, the frequency of occurrence and the codon distribution frequency of the considered amino acid play a role.

\section{Conclusions}

It is believed that the mutations are essentially random effects, especially in the non coding seuqences. For the coding sequences it is known the presence of evolutionary bias. Our analysis concerns only the coding sequences and provides indication of the presence of general pattern and symmetry, not before observed. By trial and errors, following the leading idea to incorporate in a suitable metric in a n-dim. space the effects of the near neighbours and the influence of the physico-chemical properties of the different amino acids in the rate mutation, we have build a simple model which is able to reproduce in a semi-quantitative way the hierarchy of the most frequently observed mutation between amino acids. The predictions well agree with the experimental data of PET91. One should check that no inconsistency appears in the computed inequalities. This is true for the reported set, but it has to be carefully checked for all the mutations rates. It should also be noticed that the model is able to explain some puzzling features, for example:

1. the almost equality of the rates $R(G l y \Leftrightarrow A s p)$ and $R(G l y \Leftrightarrow A r g)$ (Exp.: 70), the first mutation resulting from the transition of the 1st nucleotide, $G G R \Leftrightarrow A G R$, and the second from the transitions of the 2 rd nucleotide, $G G R \Leftrightarrow G A R$; 
2. the fact that $R(G l n \Leftrightarrow H i s)(C A R \Leftrightarrow C A Y$, transversion of the 3rd nucleotide) is lower than $R(G l n \Leftrightarrow G l u)(C A R \Leftrightarrow G A R$, transversion of the 1st nucleotide)

3. the fact that $R(\operatorname{Ser} \Leftrightarrow T h r)$ is lower than $R(\operatorname{Ser} \Leftrightarrow A l a)$ although any codon of the sextet Ser can go into the multiplet encoding $T h r$ by single nucleotide change while only the codons of the quartet UCN can go into the multiplet encoding Ala, by single nucleotide change.

A more quantitative analysis requires to take into account the normalisation of the transition matrix

$$
\sum_{j} Q_{j i}=1 \quad \forall i
$$

and to evaluate the function $F$ of eq.(8) . Moreover one should know the codon usage frequency. The parametrization in terms of only 4 parameters (which indeed can be reduced to 3 as one can be absorbed in the function $F$ ) and the identification of a codon with a real number may be a too simple choice. Going on with the analysis, likely, one will face some inconsistencies between the theoretical relations. Hopefully these pathologies can be cured with slight modifications of eqs.(8) and (10).

It is appropriate to underline that this approach can be easily generalized to describe more complex phenomena, neglected in this paper, as the multiple nucleotide changes, the observed presence of hotspots for the mutations, the variation of the mutations with the type of proteins, the probable occurrence of spurts in the evolution, the scaling behavior of the mean parameter substitution in function of the total length of genome [14], etc. A criticism can be raised against this model: it is essentially based on the properties of the genetic code while the accepted mutations are the replacement of an amino acid by a similar one. Some of the chemical properties which mostly influence the chances of mutations, like the hydrophobicity, charge, size, are related to the genetic code, [10, but many of the physical chemical properties of the amino acids are believed to have been more imposed by natural selection than by genetic code constraints. If the plausibility of the model is confirmed, this arises a puzzling question. The comparison for the mutation rates between the predicted values of the theoretical time evolution operator $\mathbf{P}(\mathbf{t})$ and the experimental values of the evolution distance matrix M, which can be criticized from many points of view, has been done as the amino acid mutation matrix is, at my knowledge, the only source of mutation data with a large statistics, obtained by analysing many thousands of proteins.

\section{References}

[1] G. Gamow, Possible Relation between Deoxyribonucleic Acid and Protein Structures, Nature 173, (1954), 318.

[2] M. Singer, P. Berg, Genes and Genomes, Editions Vigot, Paris (1992).

[3] Rodriguez F., Oliver J.L., Marin A. and Medina J.R., The General Stochastic Model for Nucleotide Substitution, J.Theor.Biol. 142, (1990), 485.

[4] Wen-Hsiung Li, Molecular Evolution, Sinauer Associates Incorporated (Sunderland, 1997).

[5] R.D. Blake, S.T. Hess and J. Nicholson-Tuell, The Influence of Nearest Neighbors on the Rate and Pattern of Spontaneous Point Mutations, J.Mol.Evol. 34, (1992), 189. 
[6] Arndt Peter F., Burge Christopher B. and Hwa Terence, DNA Sequence Evolution with Neighbor-Dependent Mutation, J.Comput.Biol. 10 (2003) 313.

[7] B.G. Konopel'chenko, Yu.B. Rumer, Classification of Codons in the Genetic Code, Translated from Doklady Akademi Nauk SSSR 223, N.2, (1975) 471.

[8] Frappat L., Sciarrino A., Sorba P.,A crystal base for the genetic code, Phys. Lett. A 250 (1998) 214.

[9] M. Kashiwara, Crystalizing the q-analogue of universal enveloping algebras, Commun. Math. Phys. 133 (1990), 249.

[10] Frappat L., Sciarrino A., Sorba P.,Predictions of Physical-Chemical Properties of Amino Acids from Genetic Code, J.Biol. Phys. 28 (2002) 17.

[11] M.O. Dayhoff, R.M. Schwartz and B.C. Orcutt, in Atlas of Protein Sequence and Structure, National Biomedical Research Foundation, Washigton D.C, Vol. 5, Suppl. 3, 345 (1978).

[12] Jones D.T., Taylor W.R. and Thornton J.M., The rapid generation of mutation data matrix from protein sequences, CABIOS 8, (1992), 275.

[13] Henikoff S. and Henikoff J.G., Amino acid substitution matrices from protein blocks, Proc.Natl.Acad.Sci USA 89, (1992), 10915.

[14] Nilsson M. and Snoad N., Optimal Mutation Rates in Dynamic Environments, physics/0004042. 
Table 3: The eukaryotic or standard code code. Upper labels denote different irreps.

\begin{tabular}{|c|c|c|c|c|c|c|c|c|c|c|c|}
\hline codon & amino acid & $J_{H}$ & $J_{V}$ & $J_{3, H}$ & $J_{3, V}$ & codon & amino acid & $J_{H}$ & $J_{V}$ & $J_{H, 3}$ & $J_{V, 3}$ \\
\hline $\mathrm{CCC}$ & Pro P & $3 / 2$ & $3 / 2$ & $3 / 2$ & $3 / 2$ & UCC & Ser S & $3 / 2$ & $3 / 2$ & $1 / 2$ & $3 / 2$ \\
\hline $\mathrm{CCU}$ & Pro P & $(1 / 2$ & $3 / 2)^{1}$ & $1 / 2$ & $3 / 2$ & $\mathrm{UCU}$ & Ser S & $(1 / 2$ & $3 / 2)^{1}$ & $-1 / 2$ & $3 / 2$ \\
\hline CCG & Pro P & $(3 / 2$ & $1 / 2)^{1}$ & $3 / 2$ & $1 / 2$ & UCG & Ser $\mathrm{S}$ & $(3 / 2$ & $1 / 2)^{1}$ & $1 / 2$ & $1 / 2$ \\
\hline $\mathrm{CCA}$ & Pro P & $(1 / 2$ & $1 / 2)^{1}$ & $1 / 2$ & $1 / 2$ & $\mathrm{UCA}$ & Ser $\mathrm{S}$ & $(1 / 2$ & $1 / 2)^{1}$ & $-1 / 2$ & $1 / 2$ \\
\hline CUC & Leu L & $(1 / 2$ & $3 / 2)^{2}$ & $1 / 2$ & $3 / 2$ & UUC & Phe F & $3 / 2$ & $3 / 2$ & $-1 / 2$ & $3 / 2$ \\
\hline CUU & Leu L & $(1 / 2$ & $3 / 2)^{2}$ & $-1 / 2$ & $3 / 2$ & UUU & Phe F & $3 / 2$ & $3 / 2$ & $-3 / 2$ & $3 / 2$ \\
\hline CUG & Leu L & $(1 / 2$ & $1 / 2)^{3}$ & $1 / 2$ & $1 / 2$ & UUG & Leu L & $(3 / 2$ & $1 / 2)^{1}$ & $-1 / 2$ & $1 / 2$ \\
\hline CUA & Leu L & $(1 / 2$ & $1 / 2)^{3}$ & $-1 / 2$ & $1 / 2$ & UUA & Leu L & $(3 / 2$ & $1 / 2)^{1}$ & $-3 / 2$ & $1 / 2$ \\
\hline CGC & $\overline{A r g} \mathrm{R}$ & $(3 / 2$ & $1 / 2)^{2}$ & $3 / 2$ & $1 / 2$ & $\overline{\mathrm{UGC}}$ & Cys C & $(3 / 2$ & $1 / 2)^{2}$ & $1 / 2$ & $1 / 2$ \\
\hline $\mathrm{CGU}$ & Arg R & $(1 / 2$ & $1 / 2)^{2}$ & $1 / 2$ & $1 / 2$ & UGU & Cys C & $(1 / 2$ & $1 / 2)^{2}$ & $-1 / 2$ & $1 / 2$ \\
\hline CGG & Arg R & $(3 / 2$ & $1 / 2)^{2}$ & $3 / 2$ & $-1 / 2$ & UGG & Trp W & $(3 / 2$ & $1 / 2)^{2}$ & $1 / 2$ & $-1 / 2$ \\
\hline CGA & Arg R & $(1 / 2$ & $1 / 2)^{2}$ & $1 / 2$ & $-1 / 2$ & UGA & Ter & $(1 / 2$ & $1 / 2)^{2}$ & $-1 / 2$ & $-1 / 2$ \\
\hline$\overline{\mathrm{CAC}}$ & His $\mathrm{H}$ & $(1 / 2$ & $1 / 2)^{4}$ & $1 / 2$ & $1 / 2$ & $\overline{\mathrm{UAC}}$ & Tyr Y & $(3 / 2$ & $1 / 2)^{2}$ & $-1 / 2$ & $1 / 2$ \\
\hline $\mathrm{CAU}$ & His $\mathrm{H}$ & $(1 / 2$ & $1 / 2)^{4}$ & $-1 / 2$ & $1 / 2$ & UAU & Tyr Y & $(3 / 2$ & $1 / 2)^{2}$ & $-3 / 2$ & $1 / 2$ \\
\hline CAG & Gln Q & $(1 / 2$ & $1 / 2)^{4}$ & $1 / 2$ & $-1 / 2$ & UAG & Ter & $(3 / 2$ & $1 / 2)^{2}$ & $-1 / 2$ & $-1 / 2$ \\
\hline CAA & Gln Q & $(1 / 2$ & $1 / 2)^{4}$ & $-1 / 2$ & $-1 / 2$ & UAA & Ter & $(3 / 2$ & $1 / 2)^{2}$ & $-3 / 2$ & $-1 / 2$ \\
\hline GCC & Ala A & $3 / 2$ & $3 / 2$ & $3 / 2$ & $1 / 2$ & $\overline{\mathrm{ACC}}$ & Thr T & $3 / 2$ & $3 / 2$ & $1 / 2$ & $1 / 2$ \\
\hline $\mathrm{GCU}$ & Ala A & $(1 / 2$ & $3 / 2)^{1}$ & $1 / 2$ & $1 / 2$ & $\mathrm{ACU}$ & Thr T & $(1 / 2$ & $3 / 2)^{1}$ & $-1 / 2$ & $1 / 2$ \\
\hline GCG & Ala A & $(3 / 2$ & $1 / 2)^{1}$ & $3 / 2$ & $-1 / 2$ & ACG & Thr T & $(3 / 2$ & $1 / 2)^{1}$ & $1 / 2$ & $-1 / 2$ \\
\hline GCA & Ala A & $(1 / 2$ & $1 / 2)^{1}$ & $1 / 2$ & $-1 / 2$ & $\mathrm{ACA}$ & Thr T & $(1 / 2$ & $1 / 2)^{1}$ & $-1 / 2$ & $-1 / 2$ \\
\hline$\overline{\text { GUC }}$ & $\overline{\text { Val V }}$ & $(1 / 2$ & $3 / 2)^{2}$ & $1 / 2$ & $1 / 2$ & $\overline{\mathrm{AUC}}$ & Ile I & $3 / 2$ & $3 / 2$ & $-1 / 2$ & $1 / 2$ \\
\hline GUU & Val V & $(1 / 2$ & $3 / 2)^{2}$ & $-1 / 2$ & $1 / 2$ & AUU & Ile I & $3 / 2$ & $3 / 2$ & $-3 / 2$ & $1 / 2$ \\
\hline GUG & Val V & $(1 / 2$ & $1 / 2)^{3}$ & $1 / 2$ & $-1 / 2$ & AUG & Met M & $(3 / 2$ & $1 / 2)^{1}$ & $-1 / 2$ & $-1 / 2$ \\
\hline GUA & Val V & $(1 / 2$ & $1 / 2)^{3}$ & $-1 / 2$ & $-1 / 2$ & AUA & Ile I & $(3 / 2$ & $1 / 2)^{1}$ & $-3 / 2$ & $-1 / 2$ \\
\hline$\overline{G G C}$ & Gly G & $3 / 2$ & $3 / 2$ & $3 / 2$ & $-1 / 2$ & $\overline{\mathrm{AGC}}$ & Ser S & $3 / 2$ & $3 / 2$ & $1 / 2$ & $-1 / 2$ \\
\hline GGU & Gly G & $(1 / 2$ & $3 / 2)^{1}$ & $1 / 2$ & $-1 / 2$ & AGU & Ser S & $(1 / 2$ & $3 / 2)^{1}$ & $-1 / 2$ & $-1 / 2$ \\
\hline GGG & Gly G & $3 / 2$ & $3 / 2$ & $3 / 2$ & $-3 / 2$ & AGG & $\operatorname{Arg} R$ & $3 / 2$ & $3 / 2$ & $1 / 2$ & $-3 / 2$ \\
\hline GGA & Gly G & $(1 / 2$ & $3 / 2)^{1}$ & $1 / 2$ & $-3 / 2$ & AGA & $\operatorname{Arg} R$ & $(1 / 2$ & $3 / 2)^{1}$ & $-1 / 2$ & $-3 / 2$ \\
\hline$\overline{\text { GAC }}$ & Asp D & $(1 / 2$ & $3 / 2)^{2}$ & $1 / 2$ & $-1 / 2$ & $\overline{\mathrm{AAC}}$ & Asn N & $3 / 2$ & $3 / 2$ & $-1 / 2$ & $-1 / 2$ \\
\hline GAU & $\mathrm{D}$ & $(1 / 2$ & $3 / 2)^{2}$ & $-1 / 2$ & $-1 / 2$ & AAU & Asn N & $3 / 2$ & $3 / 2$ & $-3 / 2$ & $-1 / 2$ \\
\hline GAG & Glu E & $(1 / 2$ & $3 / 2)^{2}$ & $1 / 2$ & $-3 / 2$ & $\mathrm{AAG}$ & Lys K & $3 / 2$ & $3 / 2$ & $-1 / 2$ & $-3 / 2$ \\
\hline GAA & Glu E & $(1 / 2$ & $3 / 2)^{2}$ & $-1 / 2$ & $-3 / 2$ & $\mathrm{AAA}$ & Lys K & $3 / 2$ & $3 / 2$ & $-3 / 2$ & $-3 / 2$ \\
\hline
\end{tabular}


Table 4: Theoretical inequalities for the rate mutations between two couples of amino acids. In the last two columns the experimental rate, from [12, for each couple.

\begin{tabular}{c|rc}
\hline Theor: Rate $(\mathrm{I})<$ Rate $(\mathrm{II})$ & Exp-I & Exp-II \\
\hline$R($ Asp $\Leftrightarrow$ Ala $)<R($ Glu $\Leftrightarrow$ Ala $)$ & 63 & 82 \\
$R($ His $\Leftrightarrow$ Pro $)<R($ Gln $\Leftrightarrow$ Pro $)$ & 58 & 81 \\
$R($ Gly $\Leftrightarrow$ Arg $)<R($ Gly $\Leftrightarrow$ Ser $)$ & 70 & 129 \\
$R($ Gly $\Leftrightarrow$ Asp $)<\approx R($ Gly $\Leftrightarrow$ Glu $)$ & 66 & 70 \\
$R($ Trp $\Leftrightarrow$ Arg $<\approx R($ Met $\Leftrightarrow$ Thr $)$ & 7 & 123 \\
$R($ Gly $\Leftrightarrow$ Arg $)<\approx R($ Gly $\Leftrightarrow$ Glu $)$ & 70 & 70 \\
$R($ Gln $\Leftrightarrow$ Arg $)<R($ His $\Leftrightarrow$ Arg $)$ & 154 & 164 \\
$R($ Asn $\Leftrightarrow$ Asp $)<R($ Asn $\Leftrightarrow$ Ser $)$ & 284 & 344 \\
$R($ Lys $\Leftrightarrow$ Gln $)<R($ Asn $\Leftrightarrow$ His $)$ & 122 & 150 \\
$R($ Lys $\Leftrightarrow$ Arg $)<R($ Asn $\Leftrightarrow$ Ser $)$ & 334 & 344 \\
$R($ Ala $\Leftrightarrow$ Thr $)<R($ Ala $\Leftrightarrow$ Ser $)$ & 267 & 284 \\
$R($ Met $\Leftrightarrow$ Thr $)<R($ Met $\Leftrightarrow$ Val $)$ & 123 & 201 \\
$R($ Tyr $\Leftrightarrow$ Asp $)<R($ Tyr $\Leftrightarrow$ Ser $)$ & 23 & 43 \\
$R($ Tyr $\Leftrightarrow$ Ser $)<R($ Tyr $\Leftrightarrow$ His $)$ & 43 & 134 \\
$R($ Val $\Leftrightarrow$ Leu $)<R($ Val $\Leftrightarrow$ Ala $)$ & 161 & 226 \\
$R($ Val $\Leftrightarrow$ Ala $)<R($ Val $\Leftrightarrow$ Ile $)$ & 226 & 504 \\
$R($ Ser $\Leftrightarrow$ Thr $)<R($ Ser $\Leftrightarrow$ Ala $)$ & 278 & 297 \\
$R($ Pro $\Leftrightarrow$ Thr $)<R($ Pro $\Leftrightarrow$ Leu $)$ & 69 & 97 \\
$R($ Pro $\Leftrightarrow$ Thr $)<R($ Ser $\Leftrightarrow$ Ala $)$ & 69 & 297 \\
$R($ Pro $\Leftrightarrow$ Ala $)<R($ His $\Leftrightarrow$ Arg $)$ & 150 & 164 \\
$R($ Ile $\Leftrightarrow$ Thr $)<R($ His $\Leftrightarrow$ Arg $)$ & 149 & 164 \\
$R($ His $\Leftrightarrow$ Arg $)<R($ Pro $\Leftrightarrow$ Ser $)$ & 164 & 190 \\
$R($ Thr $\Leftrightarrow$ Ile $)<R($ Thr $\Leftrightarrow$ Ser $)$ & 134 & 325 \\
\hline
\end{tabular}

